\title{
Widely Tunable Wavelength Exchange in Anomalous-Dispersion Regime
}

\author{
Rebecca W. L. Fung, Student Member, IEEE, Henry K. Y. Cheung, Student Member, IEEE, and
}

Kenneth K. Y. Wong, Member, IEEE

\begin{abstract}
We demonstrate the $10-\mathrm{Gb} / \mathrm{s}$ wavelength exchange with two pumps in the anomalous-dispersion region. Results show that performance degradation caused by Raman gain is avoided so that a nearly complete exchange can be achieved. In this letter, we also investigate the tunability of wavelength exchange for the first time to the best of our knowledge. Complete wavelength exchange is achieved with tuning range $>15 \mathrm{~nm}$. A bit-error rate of $<10^{-9}$ is maintained with power penalties of $\sim 2 \mathrm{~dB}$ using $2^{7}-1$ pseudorandom bit sequence (PRBS).
\end{abstract}

Index Terms-Four-wave mixing (FWM), optical parametric amplification (OPA), stimulated Raman scattering (SRS) , wavelength exchange (WE).

\section{INTRODUCTION}

W AVELENGTH EXCHANGE (WE) relies on four-wave mixing (FWM) phenomenon in highly nonlinear dispersion-shifted fibers (HNL-DSF), and it has been extensively studied in recent research [1], [2]. Simultaneous conversion of two signals can be achieved by a suitable choice of an asymmetric frequency assignment [3] wherein two pumps ( $\omega_{1}$ and $\left.\omega_{2}\right)$ are symmetric to the signal and idler $\left(\omega_{s}\right.$ and $\left.\omega_{i}\right)$ with respect to the zero-dispersion frequency $\omega_{0}$, that is, $\omega_{1}+\omega_{i}=$ $\omega_{2}+\omega_{s}=2 \omega_{0}$ [4]. Previously, simultaneous swapping between two signals could be achieved by a suitable choice of wavelengths of two pumps in the normal dispersion region and the two signals in the anomalous region with respect to the zero-dispersion frequency $\omega_{0}$ of a fiber (denoted as WE I) [1]. Complete WE can be obtained for any initial phase difference. The process can be regarded as phase insensitive if the exchange is complete. However, the phase-sensitive operation can be a problem if the WE is not complete [1]. Past results showed that the pump-induced Raman amplification introduced asymmetric power transfer that degraded the performance of the WE I process. Such performance degradation was particularly severe when the two pumps were arranged orthogonally in the normal dispersion region [5]. Therefore, we have recently proposed another configuration with two pumps in the anomalous dispersion regime (denoted as WE II), where the performance degradation caused by Raman gain can be suppressed significantly [6]. In theory, no Raman gain is provided by pumps allocated in anomalous dispersion region. Therefore, it is expected that

Manuscript received May 8, 2007; revised July 25, 2007. This work was supported in part by a grant from the Research Grants Council of the Hong Kong Special Administrative Region, China (Project HKU 7179/06E).

The authors are with the Department of Electrical and Electronic Engineering, the University of Hong Kong, Hong Kong (e-mail: wlfung@eee.hku.hk).

Color versions of one or more of the figures in this letter are available online at http://ieeexplore.ieee.org.

Digital Object Identifier 10.1109/LPT.2007.906826

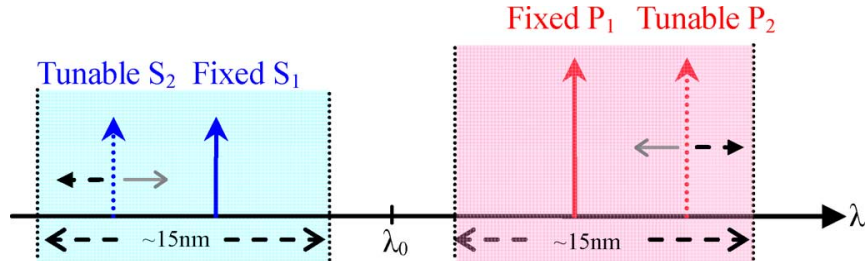

Fig. 1. Tuning operation of WE II.

power transfer asymmetry can be avoided in WE II [6]. With this arrangement, signals in the normal dispersion region exhibit symmetric power transfer characteristics and a nearly complete wavelength exchange can be achieved.

In this letter, we demonstrate an experimental validation of WE II with two $10-\mathrm{Gb} / \mathrm{s} 2^{7}-1$ pseudorandom bit sequence (PRBS) signals. The performance of the exchanger is quantified by the measurements of eye diagrams and bit-error rate (BER).

Moreover, we also investigate the tuning range of WE II in the anomalous-dispersion region in this letter. Previous research works have demonstrated the tunability of wavelength conversion based on FWM [7], [8]. In theory, the exchanged wavelengths can be arbitrary by simply tuning the pump wavelength(s) correspondingly, which has not been investigated in literature. Therefore, we investigate the tuning range of WE II by fixing one pump wavelength $\left(\mathrm{P}_{1}\right)$ while tuning another pump $\left(\mathrm{P}_{2}\right)$, both in the anomalous dispersion region, as depicted in Fig. 1. One signal $\left(\mathrm{S}_{1}\right)$ is fixed while the other signal $\left(\mathrm{S}_{2}\right)$ is tuned according to the frequency relationship $\omega_{\mathrm{S} 1}+\omega_{\mathrm{P} 1}=\omega_{\mathrm{S} 2}+\omega_{\mathrm{P} 2}$. Result shows that WE II with tunable range of $15 \mathrm{~nm}$ is achieved. The performance of the WE II over the tuning range is also quantified by the measurement of eye diagrams and BER.

\section{EXPERIMENT}

The experimental setup is shown in Fig. 2. The wavelength exchanger consisted of $1 \mathrm{~km}$ of HNL-DSF with zero-dispersion wavelength $\lambda_{0}$ of $1541 \mathrm{~nm}$, a dispersion slope of $0.03 \mathrm{ps} / \mathrm{nm}^{2} \mathrm{~km}$, and a fiber nonlinearity coefficient $\gamma$ of $12 \mathrm{~W}^{-1} \mathrm{~km}^{-1}$. The two pumps were prepared by two tunable laser sources, TLS1 and TLS2, which were fixed at $1549 \mathrm{~nm}$ and tuned from 1544 to $1558 \mathrm{~nm}$, respectively. They were phase-modulated (PM) by a 10-Gb/s $2^{7}-1$ PRBS to suppress stimulated Brillouin scattering (SBS) [9]. Erbium-doped fiber amplifier (EDFA) 1 served as the preamplifier to a booster EDFA 2. Two tunable bandpass filters (TBPF) with 2-nm bandwidth were inserted after EDFA 1 so as to filter out the two pumps separately and reduce amplified spontaneous emission (ASE) noise. Two polarization controllers (PC 3 and 4) were used to control the state of polarization ( $\mathrm{SOP}$ ) of the 


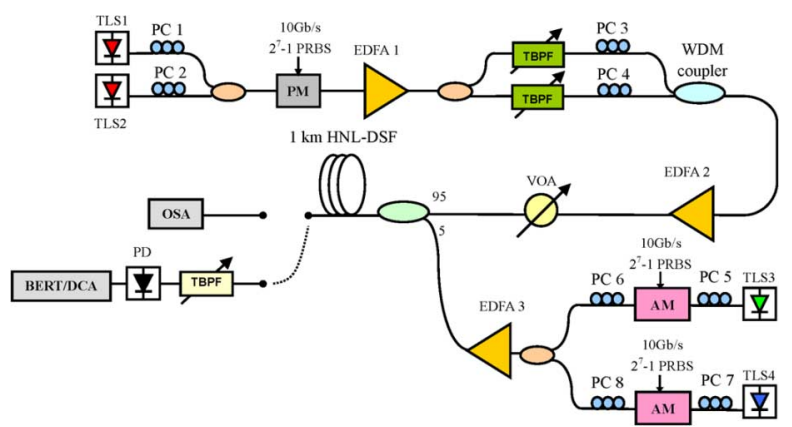

Fig. 2. Experimental setup of the wavelength exchanger.

two pumps such that orthogonal pump configuration could be maintained by minimizing the power of the spurious FWM components. The exchange efficiency was shown to be higher by using orthogonal pump allocation [1]. The two pumps were then combined with the use of a bandpass filter (BPF), which has a passband of 1547-1549.5 $\mathrm{nm}$. Wavelengths of the two signals, prepared by TLS3 and TLS4, were fixed at $1534 \mathrm{~nm}$ and tuned from 1525 to $1539 \mathrm{~nm}$, respectively. They were amplitude-modulated with a $10-\mathrm{Gb} / \mathrm{s} 2^{7}-1$ PRBS. Note that a $2^{7}-1$ PRBS was a suboptimal sequence for phase modulation (PM) to provide an efficient SBS suppression in this experiment. Since the same PRBS pattern source was used for both $\mathrm{PM}$ and amplitude modulation (AM) at the same time, a $2^{7}-1$ PRBS was chosen as the data pattern. A longer data pattern can be adopted as the fiber-based four-wave mixing process, by its nature, is a pattern independent process. PC 6 and 8 aligned the signals with the orthogonal pumps. EDFA 3, with a gain bandwidth of 1524-1560 nm, was used to compensate for the insertion loss of the AMs. A variable optical attenuator (VOA) was inserted after EDFA 2 to adjust the input pump powers; while a $95 / 5$ coupler combined the pumps and the signals into the fiber. The output power from the fiber was sent to the optical spectrum analyzer (OSA) to display the spectrum after the exchange. We then measured their waveforms and BER using the digital communication analyzer (DCA) and BER tester (BERT), respectively. A TBPF with a tunable range of $1525-1575 \mathrm{~nm}$ was used at the fiber output to filter out the exchanged signals.

\section{RESULTS AND DISCUSSION}

It has been discussed that pump-induced Raman amplification causes asymmetric power transfer in the normal dispersion region. Therefore, orthogonal WE II is introduced to improve the symmetry of WE [6]. The experimental result of orthogonal WE II is shown in Fig. 3. The experimental result demonstrates that symmetric power transfer characteristics can be obtained from WE II such that the maximum power of the idler (generated at $1529 \mathrm{~nm}$ ) can be attained together with the minimum power of the residual signal (at $1534 \mathrm{~nm}$ ). The maximum normalized idler power is equal to unity in the WE process as no Raman gain is provided by the two pumps. Result shows that a nearly complete WE can be obtained.

During the practical WE, two signals placed at a fixed wavelength of $1534 \mathrm{~nm}$ and an arbitrary wavelength, for example, at $1529 \mathrm{~nm}$, undergo exchanging process simultaneously. Under the process, the two corresponding idlers are generated at 1529 and $1534 \mathrm{~nm}$ accordingly. Conversion ratio (CR) is defined as

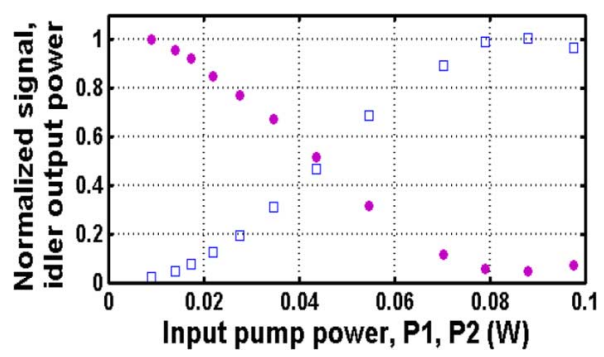

Fig. 3. WE transfer characteristics in WE II with signal at $1534 \mathrm{~nm}$ and idler at $1529 \mathrm{~nm}$ - signal power $(\bullet)$ and idler power $(\square)[3]$.

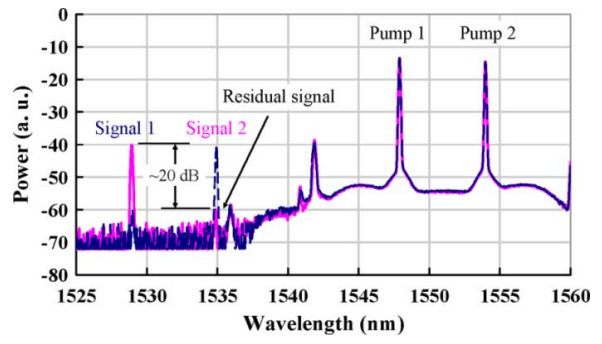

Fig. 4. Measured optical spectra after WE.

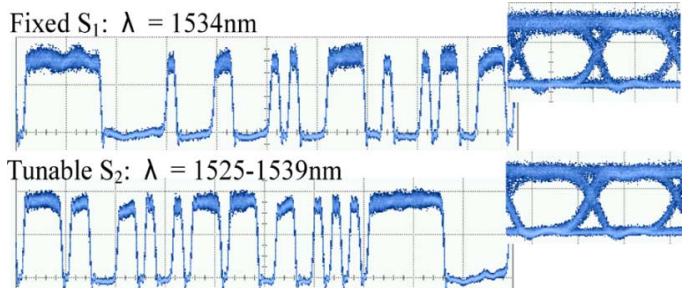

Fig. 5. Data streams before WE.

the power ratio between the maximum converted signal (idler) and the minimum residual signal [5]. Here, the CRs were measured to be $\sim 20 \mathrm{~dB}$ in both cases after WE as shown in Fig. 4. Note that a slight frequency offset of the signal helps reduce the crosstalk between the converted signal and the residual signal in practice [1].

Fig. 5 represents the original signal waveforms observed at $1534 \mathrm{~nm}$ and other tunable wavelengths $(1525-1539 \mathrm{~nm})$ before WE. The insets show their eye diagrams with $Q$-factors of 10 and $10.4 \mathrm{~dB}$ as well as extinction ratios (ERs) of 11.3 and $11.7 \mathrm{~dB}$, respectively.

The exchanged signals after WE observed at 1534 and $1529 / 1533 \mathrm{~nm}$ (with the best/worst performance over the tunable range) are chosen to be shown in Fig. 6(a) and (b). In Fig. 6(a)(i) and (b)(i), the original signal at 1529/1533 nm is exchanged to its idler wavelength at $1534 \mathrm{~nm}$, while Fig. 6(a)(ii) and (b)(ii) illustrate that the original signal at $1534 \mathrm{~nm}$ is efficiently exchanged to its corresponding idler wavelength at $1529 / 1533 \mathrm{~nm}$ indicating that WE II is successfully achieved. The insets show their eye diagrams with $Q$-factors of $9.2 / 7.93 \mathrm{~dB}$ and $9.28 / 6.6 \mathrm{~dB}$ as well as ERs of $10.32 / 8.64 \mathrm{~dB}$ and 10.7/8.71 dB, respectively. The noisy mark level is caused by the EDFA ASE noise from the two pumps. It is suggested that the ASE noise of the pumps can be suppressed by a fiber Bragg grating (FBG) with a narrow bandwidth and a high suppression level [10].

The $Q$-factors and ERs of the exchanged signals over the tuning range are recorded in Fig. 7. It shows that good performance WE can be successfully maintained over $15 \mathrm{~nm}$ 
(a) $\lambda=1534 \mathrm{~nm}, Q=9.2 \mathrm{~dB}, E R=10.32 \mathrm{~dB}$

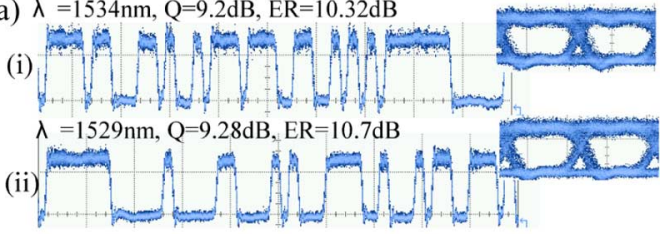

(b) $\lambda=1534 \mathrm{~nm}, Q=7.93 \mathrm{~dB}, E R=8.64 \mathrm{~dB}$

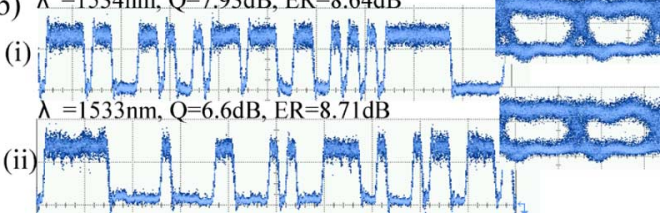

Fig. 6. Exchanged signals with (a) best and (b) worst performance over the tuning range.

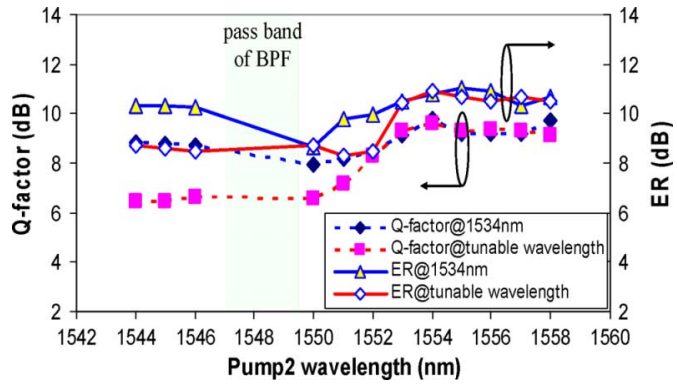

Fig. 7. $Q$-factors and ERs among exchanged signals over the tunable wavelength range.

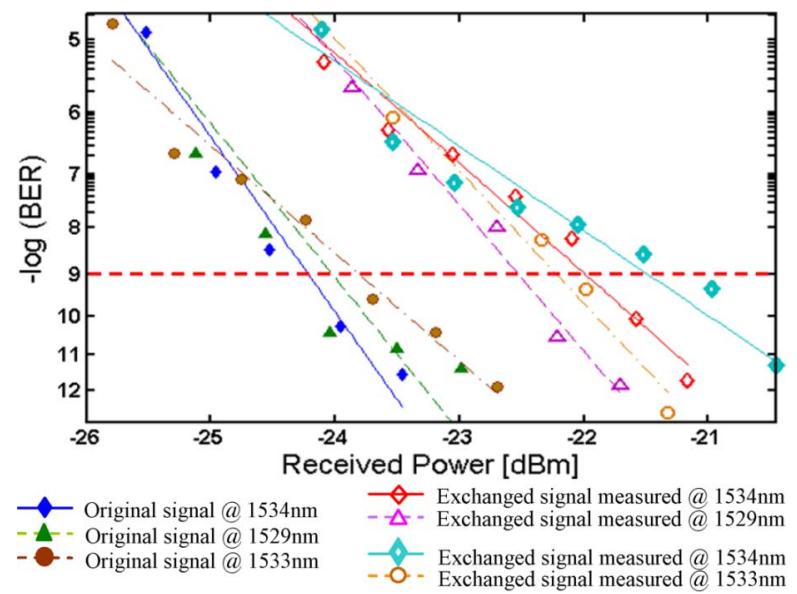

Fig. 8. Measured BER curves for the exchanged signals before and after WE.

$(1544-1559 \mathrm{~nm})$ tuning range with $Q$-factors $>6.5 \mathrm{~dB}$ and ERs of the eyes $>8.5 \mathrm{~dB}$. The $Q$-factors and ERs among the exchanged signals vary up to $\sim 3 \mathrm{~dB}$ over the $15-\mathrm{nm}$ tuning range. The tuning range is limited by the gain bandwidth of the EDFA and the tuning range of TBPF in our experiment, otherwise a wider tuning range of WE can be recorded. Moreover, different dispersion slopes of different fibers may affect the tuning range of WE, which will be investigated in the further study. It is noteworthy that the WE II performance is slightly degraded when $\mathrm{P}_{2}$ is tuned near the $\lambda_{0}$. The reason is owing to the pump-induced optical parametric amplification (OPA) on the exchanged signals near the $\lambda_{0}$ [11]. The performance is even worse when the tunable signal wavelengths are placed near the peak of OPA gain spectrum.
To evaluate the performance of WE II, the receiver sensitivities of the two exchanged signals (the best and the worst cases) are measured and compared with their corresponding original signals. The measured BER curves are plotted in Fig. 8. At BER of $10^{-9}$, the receiver sensitivities of the exchanged signals at 1534 and $1529 / 1533 \mathrm{~nm}$ are around $-22 \mathrm{dBm}$. The power penalties incurred in the exchanger are measured to be $\sim 2 \mathrm{~dB}$. This power penalty is mainly due to the phase noise introduced by the phase dithering of the pumps, and can be improved with the complementary phase dithering method [12].

\section{CONCLUSION}

We have successfully demonstrated the performance of WE and investigated the tunability of WE in the anomalous dispersion region. Results show that performance degradation caused by Raman gain is avoided so that a nearly complete WE can be achieved. The tuning range can achieve over $15 \mathrm{~nm}$ with clear eye openings observed. BERs of $<10^{-9}$ is maintained with power penalties of $\sim 2 \mathrm{~dB}$.

\section{ACKNOWLEDGMENT}

The authors would like to thank Prof. M. E. Marhic and C. H. Kwok for their comments on this letter.

\section{REFERENCES}

[1] K. Uesaka, K. K. Y. Wong, M. E. Marhic, and L. G. Kazovsky, "Wavelength exchange in a highly nonlinear dispersion-shifted fiber: Theory and experiments," IEEE J. Sel. Topics Quantum Electron., vol. 8, no. 3, pp. 560-568, May/Jun. 2002.

[2] K. K. Y. Wong, M. E. Marhic, K. Uesaka, and L. G. Kazovsky, "Demonstration of wavelength exchange in a highly-nonlinear fiber," in Eur. Conf. Opt. Commun. (ECOC), Amsterdam, The Netherlands, 2001.

[3] K. Inoue, "Tunable and selective wavelength conversion using fiber four-wave mixing with two pump lights," IEEE Photon. Technol. Lett., vol. 6, no. 12, pp. 1451-1453, Dec. 1994.

[4] M. E. Marhic, Y. Park, F. S. Yang, and L. G. Kazovsky, "Widely tunable spectrum translation and wavelength exchange by four-wave mixing in optical fibers," Opt. Lett., vol. 21, no. 23, pp. 1906-1908, 1996.

[5] R. W. L. Fung, C. H. Kwok, and K. K. Y. Wong, "Parallel and orthogonal wavelength exchange," presented at the Optoelectronics Commun. Conf. (OECC), Taiwan, R.O.C., 2006, 4D2-7.

[6] R. W. L. Fung, H. K. Y. Cheung, P. P. Kuo, C. H. Kwok, and K. K. Y. Wong, "Wavelength exchange in the anomalous-dispersion regime," in Proc. Eur. Conf. Opt. Commun. (ECOC), Cannes, France, 2006.

[7] T. Tanemura, C. S. Goh, K. Kikuchi, and S. Y. Set, "Highly efficient arbitrary wavelength conversion within entire $C$-band based on nondegenerate fiber four-wave mixing," IEEE Photon. Technol. Lett., vol. 16, no. 2, pp. 551-553, Feb. 2004.

[8] C. H. Kwok, S. H. Lee, K. K. Chow, C. Shu, C. Lin, and A. Bjarklev, "Widely tunable wavelength conversion with extinction ratio enhancement using PCF-based NOLM," IEEE Photon. Technol. Lett., vol. 17, no. 12, pp. 2665-12657, Dec. 2005.

[9] S. K. Korotky, P. B. Hansen, L. Eskildsen, and J. J. Veselka, "Efficient phase modulation scheme for suppression of stimulated Brillouin scattering," in Proc. IOOC 1995, 1995, pp. 109-111, Paper WD2-1.

[10] J. L. Blows and S. E. French, "Low-noise-figure optical parametric amplifier with a continuous-wave frequency-modulated pump," Opt. Lett., vol. 27, no. 7, pp. 491-493, 2002.

[11] H. K. Y. Cheung, R. W. L. Fung, P. P. Kuo, C. H. Kwok, and K. K. Y. Wong, "Effect of stimulated Raman scattering and optical parametric amplification on wavelength exchange," presented at the IEEE TENCON, Hong Kong, China, 2006, Paper OP1.1.

[12] K. K. Y. Wong, M. E. Marhic, and L. G. Kazovsky, "Phase-conjugate pump dithering for high-quality idler generation in a fiber optical parametric amplifier," IEEE Photon. Technol. Lett., vol. 15, no. 1, pp. 33-35, Jan. 2003. 